\title{
Hedging Stock Options Using Futures Contracts on the Stock
}

\author{
Mihai Grigore Bunea Domsa \\ Department of Mathematics and Computer Science, Babes-Bolyai University, Cluj-Napoca, Romania
}

Email address:

mihai.bunea@math.ubbcluj.ro

\section{To cite this article:}

Mihai Grigore Bunea Domsa. Hedging Stock Options Using Futures Contracts on the Stock. Applied and Computational Mathematics. Vol. 4, No. 3, 2015, pp. 214-219. doi: 10.11648/j.acm.20150403.24

\begin{abstract}
The aim of this paper is to present the price and replicating strategy for an European option on spot (or cash) underlier with continuous dividend yield, when the instrument used in the dynamic hedging of the option is a futures contract on the respective underlier. It formalizes the heuristic practice among option traders to replicate options on a stock index using futures on the respective stock index and investigates weather the obtained results differ significantly from what they would get using the actual stock index, as required by Black-Scholes pricing. Heuristically, the substitution is supported by index and futures prices being close, at least for small dividends and time to maturity. Our method is to express this practice in accounting terms, derive the self-financing portfolio dynamics and then the closed form option price and delta. Finally, run numerical simulations and compare results obtained by Black-Scholes versus our approach. Results show both the price and delta formulas differ from Black-Scholes, however numeric simulation doesn't yield high enough differences to warrant obvious arbitrage, meaning that while not rigorously exact, the approximation is good enough for most practical use cases.
\end{abstract}

Keywords: Options Pricing, Options Replication Using Futures, Arbitrage

\section{Introduction}

The classical Black-Scholes model provides a price and a way to replicate options on a stock or stock index. As an analogy think options are bread and the stock is wheat. The amount of money one asks for a bread is what pricing is about, the quantity of wheat used to make that bread is what replication means.

A stock index is harder to buy/sell directly so traders use futures on it instead. It's like since there's no wheat, they buy flour. This raises two problems which are addressed in this paper:

(1) Is the option price the same if replicated using stocks or futures? (Is the bread price the same, weather made from wheat or from flour?)

(2) Is the replication strategy the same using stocks or using futures? (Are the baking quantities the same, weather using wheat or flour?)

\section{Notation}

- $t$ : current time;

- $S$ : a stock or stock index with current price $S(t)$ and dividend yield $q$

- $A$ : an option on the stock or stock index with current fair value $A(t)$ and expiration time $T_{a} \geq t$;

- $F$ : a future on the stock or stock index with current price $F(t)$ and expiration time $T_{f} \geq T_{a}$;

- $B$ : a bank account with current amount $B(t)$ and interest rate $r$;

- $m$ : margin factor, the percent of stock value which needs to be deposited as margin for the purchase of one future contract;

- $\Phi(x)=\frac{1}{\sqrt{2 \pi}} \int_{-\infty}^{x} e^{-\frac{t^{2}}{2}} d t$ : standard normal cumulative distribution function;

- $\varphi(x)=\frac{1}{\sqrt{2 \pi}} e^{-\frac{x^{2}}{2}}:$ standard normal probability density function.

Our goal is to formulate the option price and delta in terms of this notation.

\section{Results}

The Black-Scholes model is used to price an option when 
the underlier is a stock or stock index, see e.g. [4, Options on Stock Indexes on page 4]. In our paper we'll replicate the option using a future on the stock or stock index and we'll refer to this case as 'a new approach', shorthanded as NA. In what follows we present the differences in price and delta between Black-Scholes and the NA.

\subsection{Pricing}

The Black-Scholes price is

$$
A(t)=s f(t) e^{-r T} \Phi\left(s d_{1}\right)-s K e^{-r T} \Phi\left(s d_{2}\right)
$$

where

$$
f(t)=S(t) e^{(r-q) T}, d_{1}=\frac{\ln \frac{f(t)}{K}+\frac{\sigma^{2}}{2} T}{\sigma \sqrt{T}}
$$

The NA price is

$$
A(t)=s f(t) \Phi\left(s d_{1}\right)-s K e^{-r m T} \Phi\left(s d_{2}\right)
$$

where

$$
f(t)=F(t) e^{-(r-q)\left(T_{f}-T_{a}\right)}, d_{1}=\frac{\ln \frac{f(t)}{K}+\left(r m+\frac{\sigma^{2}}{2}\right) T}{\sigma \sqrt{T}}
$$

In both cases

$$
d_{2}=d_{1}-\sigma \sqrt{T}, T=T_{a}-t, s=\left\{\begin{array}{ll}
+1 & \text { if call option } \\
-1 & \text { if put option }
\end{array} .\right.
$$

It can be shown that the forward price $f(t)$ is the same in both formulas, therefore they differ by means of the interest rate and are only equal if the interest rate is zero. Below are numerical examples for a call option when $S=100$, $K=100, T=30$ days, $q=5 \%, \sigma=20 \%$ and interest rates of 0 , respectively $12 \%$ per year. One can see that for non zero interest rate prices are not the same, although they might be close.

\begin{tabular}{l|c|c} 
& Black-Scholes & NA \\
\hline$r=0 \%$ & 2.0833 & 2.0833 \\
\hline$r=12 \%$ & 2.5683 & 2.5938
\end{tabular}

\subsection{Replication}

As known/shown in the proof section, replicating an option is done by trading a quantity of spot or futures equal to option's delta. The analytical expression of delta is

$$
\Delta(t)= \begin{cases}\frac{\partial A(t)}{\partial S}=s e^{-q\left(T_{a}-t\right)} \Phi\left(s d_{1}\right) & \text { if Black-Scholes } \\ \frac{\partial A(t)}{\partial F}=s e^{-(r-q)\left(T_{f}-T_{a}\right)} \Phi\left(s d_{1}\right) & \text { if NA }\end{cases}
$$

Below are numerical values for the parameters from the pricing section. One can see that quantities are not the same. It is outside the scope of this paper to say what happens if one replicates an option using the wrong quantity (ex: trade futures using Black-Scholes quantity), intuitively one can guess that the result will have some error in it, however the magnitude of the error it's not straightforward.

\begin{tabular}{l|c|c} 
& Black-Scholes & NA \\
\hline$r=0 \%$ & 0.4809 & 0.4868 \\
\hline$r=12 \%$ & 0.5491 & 0.5450
\end{tabular}

\section{Proofs}

\subsection{Model}

The stock dynamics is described by a geometric Brownian motion with growth rate $\mu$, volatility $\sigma$ and Wiener process $W(t)$. The equation is

$$
\frac{d S(t)}{S(t)}=\mu d t+\sigma d W_{P}(t)
$$

The stock offers dividends modeled by a continuous dividend yield $q$ with dynamics over time described by the equation

$$
d D(t)=q S(t) d t
$$

The bank account grows at a continuously compounded interest rate $r$

$$
\frac{d B(t)}{B(t)}=r d t
$$

Future and spot price are linked by the no-arbitrage equation

$$
F(t)=S(t) e^{(r-q)\left(T_{f}-t\right)}
$$

The payout for an European call option with strike $K$ is

$$
A\left(T_{a}, S\left(T_{a}\right)\right)=\left\{\begin{array}{lll}
S\left(T_{a}\right)-K, & \text { if } & S\left(T_{a}\right)>K \\
0, & \text { if } & S\left(T_{a}\right) \leq K .
\end{array}\right.
$$

The payout for an European put option with strike $K$ is

$$
A\left(T_{a}, S\left(T_{a}\right)\right)= \begin{cases}K-S\left(T_{a}\right), & \text { if } \quad S\left(T_{a}\right)<K \\ 0, & \text { if } \quad S\left(T_{a}\right) \geq K .\end{cases}
$$

\subsection{Pricing}

The price of the option depends on the type of asset used to replicate it's payout. When that asset is the stock, the price is given by the well-known Black-Scholes formula and the replicating strategy by continuous dynamic delta-hedging in the undelier asset, see e.g. [8, Delta Hedging on page 344] . When that asset is the future, the price formula is different and 
we will deduce it in the following.

By Itö lemma, see e.g. [11, Theorem 4.10 on page 47], the dynamics of the future price has the form

$$
\begin{gathered}
d F(t, S(t))=\left(\frac{\partial F(t, S(t))}{\partial t}+\mu S(t) \frac{\partial F(t, S(t))}{\partial S}\right. \\
\left.+\frac{\sigma^{2} S(t)^{2}}{2} \frac{\partial^{2} F(t, S(t))}{\partial S^{2}}\right) d t+\sigma S(t) \frac{\partial F(t, S(t))}{\partial S} d W(t) .
\end{gathered}
$$

We have

$$
\begin{gathered}
\frac{\partial F(t, S(t))}{\partial t}=(q-r) S(t) e^{(r-q)\left(T_{f}-t\right)}, \frac{\partial F(t, S(t))}{\partial S}=e^{(r-q)\left(T_{f}-t\right)}, \\
\frac{\partial^{2} F(t, S(t))}{\partial S^{2}}=0 .
\end{gathered}
$$

Therefore,

$$
\begin{aligned}
d F(t, S(t)) & =\left((q-r) S(t) e^{(r-q)\left(T_{f}-t\right)}+\mu S(t) e^{(r-q)\left(T_{f}-t\right)} d t\right. \\
+ & \sigma S(t) e^{(r-q)\left(T_{f}-t\right)} d W(t) \Rightarrow \\
d F(t, S(t)) & =((q-r) F(t)+\mu F(t) d t+\sigma F(t) d W(t) \\
& =(\mu-r+q) F(t) d t+\sigma F(t) d W(t) .
\end{aligned}
$$

Denoting $\mu-r+q=\alpha$, we have

$$
\frac{d F(t)}{F(t)}=\alpha d t+\sigma d W(t)
$$

Consequently the equation describing the dynamics of the future instrument is similar to the one describing the stock, where the growth rate of the stock $\mu$ was replaced by a growth rate of the future $\alpha$ but the volatility $\sigma$ and driving Wiener process $W(t)$ are the same.

The key in pricing the option is specifying it's replicating portfolio. A replicating portfolio is a series of trades in the future instrument financed by a bank account. It's value at a moment in time is

$$
R(t)=h(t) F(t)-B(t),
$$

where $B(t)$ is the amount of money borrowed from the bank to buy a quantity of futures $h(t)$.

The purpose of a replicating portfolio is to exactly follow (replicate) the price of the option. At any moment in time we want to have the static relation $R(t)=A(t)$, from which follows that it's dynamics is given by

$$
d R(t)=d A(t) \equiv h(t) d F(t)-d B(t)=d A(t) .
$$

At this point we need to observe a particularity of futures contracts compared to plain stocks. When one buys an unit amount of stock, one has to pay it's entire value. In accounting terms $S=B$, or the positive value debited by the purchase of stock is financed trough a credit of the same value from the bank account. Future contracts are different. One doesn't have to pay their entire value, only a fraction of it, known as the margin. For instance, if the stock price is 100 dollars, the buyer of a future contract on it might only have to pay some $10 \%$ or $20 \%$ of it. We will denote this margin factor by $m$.

When using a future for replication, the accounting book still has to hold, so $F=B$, or value debited equals value credited. The difference is that the bank account is split in two: a $B_{r}$ one which is subjected to interest rate and a $B_{z}$ one where there's no interest. Consequently

$$
F=B \Rightarrow m F+(1-m) F=B_{r}+B_{z} .
$$

The dynamics of the bank account is

$$
\begin{gathered}
d B=d B_{r}+d B_{z} \Rightarrow d B=B_{r} d t+B_{z} \cdot 0 \cdot d t \Rightarrow \\
d B=F m r d t=B m r d t .
\end{gathered}
$$

Therefore when using futures, the dynamics of the bank account is governed not by the original interest rate $r$ but by a reduced rate equal to the margin factor applied to the original rate: $r_{m}=r \cdot m$. Consequently we have

$$
d A(t)=h(t) d F(t)-d B(t) \Rightarrow d A(t)=h(t) d F(t)-r_{m} B(t) d t .
$$

To be concise we have omitted in the above equations the fact that $A=A(t, F(t))$, since it depends on both time and price of the future instrument. We need this form now in order to derive the dynamics of the option price, by applying Itö lemma once again.

$$
\begin{gathered}
d A(t, F(t))=\frac{\partial A(t, F(t))}{\partial F} d F(t) \\
+\left(\frac{\partial A(t, F(t))}{\partial t}+\frac{\sigma^{2} F(t)^{2}}{2} \frac{\partial^{2} A(t, F(t))}{\partial F^{2}}\right) d t .
\end{gathered}
$$

Consequently from the condition to follow the option price by it's replica we get the equations

$$
h(t) d F(t)=\frac{\partial A(t, F(t))}{\partial F} d F(t)
$$

and

$$
\left(\frac{\partial A(t, F(t))}{\partial t}+\frac{\sigma^{2} F(t)^{2}}{2} \frac{\partial^{2} A(t, F(t))}{\partial F^{2}}\right)=-r_{m} B(t)
$$

First equation determines out control variable: the quantity of futures we need to hold

$$
h(t)=\frac{\partial A(t, F(t))}{\partial F} .
$$

At any moment in time we need to hold a quantity of futures equal to the sensitivity of option price relative to the change in future price. This fixes the quantity but doesn't tell us what the option price is. For that we need the second equation, a deterministic PDE which fixes the option price. Let's get rid of $B(t)$ remembering that 


$$
\begin{aligned}
& A(t)=R(t)=h(t) F(t)-B(t) \Rightarrow \\
& B(t)=h(t) F(t)-A(t)=\frac{\partial A(t, F(t))}{\partial F} F(t)-A(t) .
\end{aligned}
$$

Replacing in (2) we get the PDE

$$
\begin{gathered}
\left(\frac{\partial A(t, F(t))}{\partial t}+\frac{\sigma^{2} F(t)^{2}}{2} \frac{\partial^{2} A(t, F(t))}{\partial F^{2}}\right) \\
+r_{m}\left(\frac{\partial A(t, F(t))}{\partial F} F(t)-A(t)\right)=0
\end{gathered}
$$

Dropping again time and underlier parameters from the option price function we obtain the PDE for the option price

$$
\frac{\partial A}{\partial t}+\frac{\sigma^{2} F^{2}}{2} \frac{\partial^{2} A}{\partial F^{2}}+r_{m} F \frac{\partial A}{\partial F}-r_{m} A=0 .
$$

In the following we will determine the analytical solution for European options. Fenyman-Kač formula, see e.g. [6, Proposition 5.6 on page 70], says that the solution to the above PDE is

$$
A(t, F(t))=e^{-r_{m} T} \mathrm{E}\left[A\left(T_{a}, F\left(T_{a}\right)\right)\right]
$$

where

$$
T=T_{a}-t, F\left(T_{a}\right)=F(t) e^{\left(r_{m}-\frac{\sigma^{2}}{2}\right) T+\sigma W\left(T_{a}\right)},
$$

as if the future price $F$ would follow a stochastic differential equation where the growth rate $\alpha$ is replaced by the interest rate $r_{m}$

$$
\frac{d F(t)}{F(t)}=r_{m} d t+\sigma d W(t)
$$

The option contract is specified in terms of stock price, we need to express it in terms of future. Denoting the discount factor at maturity time with

$$
D_{a}=e^{-(r-q)\left(T_{f}-T_{a}\right)}
$$

we have

$$
\begin{gathered}
F\left(T_{a}\right)=S\left(T_{a}\right) e^{(r-q)\left(T_{f}-T_{a}\right)} \Rightarrow \\
S\left(T_{a}\right)=F\left(T_{a}\right) e^{-(r-q)\left(T_{f}-T_{a}\right)}=F\left(T_{a}\right) D_{a} .
\end{gathered}
$$

The payout for an European call option with strike $K$ is

$$
A\left(T_{a}, F\left(T_{a}\right)\right)= \begin{cases}F\left(T_{a}\right) D_{a}-K, & \text { if } F\left(T_{a}\right)>\frac{K}{D_{a}}, \\ 0, & \text { if } F\left(T_{a}\right) \leq \frac{K}{D_{a}} .\end{cases}
$$

We have

$$
\begin{gathered}
F\left(T_{a}\right)=e^{X}, X \sim N\left(\mu_{X}, \sigma_{X}^{2}\right), \mu_{X}=\ln F(t)+\left(r_{m}-\frac{\sigma^{2}}{2}\right) T, \\
\sigma_{X}=\sigma \sqrt{T} .
\end{gathered}
$$

Therefore $F\left(T_{a}\right)$ is a lognormal random variable and it's probability density function is

$$
p(F)=\frac{1}{F \sigma_{X} \sqrt{2 \pi}} e^{-\frac{\left(\ln F-\mu_{X}\right)^{2}}{2 \sigma_{X}^{2}}} .
$$

By [6, formula 10 on page 3$]$ we have

$$
\int_{C}^{\infty} F p(F) d F=e^{\mu_{X}+\frac{\sigma_{X}^{2}}{2} \Phi\left(f_{1}\right)}
$$

where

$$
f_{1}=\frac{-\ln C+\mu_{X}+\sigma_{X}^{2}}{\sigma_{X}}
$$

and by [ 6 , formula 20 on page 8$]$ we have

$$
\int_{C}^{\infty} p(F) d F=\Phi\left(f_{2}\right)
$$

where $f_{2}=f_{1}-\sqrt{\sigma_{X}}$.

We can expand (3) into integral form and using (4) and (5) we get

$$
\begin{aligned}
& A(t, F(t))=e^{-r_{m} T} \int_{\frac{K}{D_{a}}}^{\infty}\left(F D_{a}-K\right) p(F) d F \\
& =e^{-r_{m} T} D_{a} \int_{\frac{K}{D_{a}}}^{\infty} F p(F) d F-e^{r_{m} T} K \int_{\frac{K}{D_{a}}}^{\infty} p(F) d F \\
& =F(t) D_{a} \Phi\left(d_{1}\right)-K D_{t} \Phi\left(d_{2}\right) \text {, } \\
& d_{1}=\frac{\ln \frac{F(t) D_{a}}{K}+\left(r_{m}+\frac{\sigma^{2}}{2}\right) T}{\sigma \sqrt{T}}, d_{2}=d_{1}-\sigma \sqrt{T} .
\end{aligned}
$$

In a similar way we can get the formula for an European put option.

\subsection{Replication}

To obtain the expression for option delta $\frac{\partial A(t)}{\partial F}$, take the expression of option price

$$
\begin{gathered}
A(t)=s F(t) D_{a} \Phi\left(s d_{1}\right)-s K e^{-r_{m} T} \Phi\left(s d_{2}\right), s= \begin{cases}+1 & \text { if call } \\
-1 & \text { if put, }\end{cases} \\
D_{a}=e^{-(r-q)\left(T_{f}-T_{a}\right)}, T=\left(T_{a}-t\right), \\
d_{1}=\frac{\ln \frac{F(t) D_{a}}{K}+\left(r_{m}+\frac{\sigma^{2}}{2}\right) T}{\sigma \sqrt{T}}, d_{2}=d_{1}-\sigma \sqrt{T} .
\end{gathered}
$$


We have

$$
\begin{gathered}
\frac{\partial d_{1}}{\partial F}=\frac{1}{F(t) \sigma \sqrt{T}}, \frac{\partial d_{2}}{\partial F}=\frac{\partial d_{1}}{\partial F}, \\
\frac{\partial A(t)}{\partial F}=\left(s D_{a} \Phi\left(s d_{1}\right)+s^{2} F(t) D_{a} \varphi\left(s d_{1}\right)\right) \frac{\partial d_{1}}{\partial F} \\
-s^{2} K e^{-r_{m} T} \varphi\left(s d_{2}\right) \frac{\partial d_{2}}{\partial F}, \\
s^{2}=1 \Rightarrow \frac{\partial A(t)}{\partial F}=s D_{a} \Phi\left(s d_{1}\right)+G(t),
\end{gathered}
$$

where

$$
\begin{gathered}
G(t)=F(t) D_{a} \varphi\left(s d_{1}\right) \frac{1}{F(t) \sigma \sqrt{T}}-K e^{-r_{m} T} \varphi\left(s d_{2}\right) \frac{1}{F(t) \sigma \sqrt{T}} \\
=\frac{1}{\sigma \sqrt{T}}\left(D_{a} \varphi\left(s d_{1}\right)-\frac{K}{F(t)} e^{-r_{m} T} \varphi\left(s d_{2}\right)\right) \\
=\frac{1}{\sigma \sqrt{2 \pi T}}\left(D_{a} e^{-\frac{\left(s d_{1}\right)^{2}}{2}}-\frac{K}{F(t)} e^{-r_{m} T} e^{-\frac{\left(s d_{2}\right)^{2}}{2}}\right) \\
=\frac{1}{\sigma \sqrt{2 \pi T}}\left(D_{a} e^{-\frac{d_{1}^{2}}{2}}-\frac{K}{F(t)} e^{-r_{m} T} e^{-\frac{d_{2}^{2}}{2}}\right) \\
=\frac{1}{\sigma \sqrt{2 \pi T}}\left(D_{a} e^{-\frac{d_{1}^{2}}{2}}-\frac{K}{F(t)} e^{-r_{m} T} e^{-\frac{\left(d_{1}-\sigma \sqrt{T}\right)^{2}}{2}}\right) \\
=\frac{1}{\sigma \sqrt{2 \pi T}}\left(D_{a} e^{-\frac{d_{1}^{2}}{2}}-\frac{K}{F(t)} e^{-r_{m} T} e^{-\frac{d_{1}^{2}}{2}} e^{d_{1} \sigma \sqrt{T}-\frac{\sigma^{2} T}{2}}\right) \\
=\frac{1}{\sigma \sqrt{2 \pi T}} e^{-\frac{d_{1}^{2}}{2}}\left(D_{a}-\frac{K}{F(t)} e^{-r_{m} T} e^{d_{1} \sigma \sqrt{T}-\frac{\sigma^{2} T}{2}}\right)=\frac{1}{\sigma \sqrt{2 \pi T}} e^{-\frac{d_{1}^{2}}{2}}\left(D_{a}\right. \\
\left.-\frac{K}{F(t)} e^{-r_{m} T} e^{\ln \frac{F(t) D_{a}}{K}}+\left(r_{m}+\frac{\sigma^{2}}{2}\right) T-\frac{\sigma^{2} T}{2}\right)=\frac{1}{\sigma \sqrt{2 \pi T}} e^{-\frac{d_{1}^{2}}{2}}\left(D_{a}\right. \\
\left.-\frac{K}{F(t)} \frac{F(t) D_{a}}{K} e^{-r_{m} T+r_{m} T+\frac{\sigma^{2} T}{2}-\frac{\sigma^{2} T}{2}}\right)=\frac{1}{\sigma \sqrt{2 \pi T}} e^{-\frac{d_{1}^{2}}{2}\left(D_{a}-D_{a}\right)}=0 .
\end{gathered}
$$

Therefore we are left with

$$
\frac{\partial A(t)}{\partial F}=s D_{a} \Phi\left(s d_{1}\right)=s e^{-(r-q)\left(T_{f}-T_{a}\right)} \Phi\left(s d_{1}\right) .
$$

\subsection{Conclusions}

We draw the conclusions by answering the questions from the introduction section.

(1) Is the option price the same if replicated using stocks or futures? No, it is not. There is a slight difference in price between the two strategies.

(2) Is the replication strategy the same using stocks or using futures? Again, it's not the same. The quantities of stock index and futures are different.

From a purely mathematical perspective, there's a difference in pricing which automatically leads to the hypothesis of possible arbitrage. The practice however is not so simple, differences are rather small and easily offsetted by missing a bit on volatility for instance. So while using our model to price options does result in slightly better margins, it's not a silver bullet.

\section{Short Review of Options Hedging Research}

The Black-Scholes framework for pricing and replicating options trough dynamic delta hedging is introduced in [8, John Hull] by fairly accessible mathematics (PDEs). A more advanced and popular method at the current time, martingale theory, can be found in [11, Thomas Björk]. An attempt to explain the nebulous concept of change of measure and its application on option pricing is presented in [10, Salih Neftci]. A large collection of ready-made formulas for valuation of various types of option contracts are listed in [4, Espen Haug].

Classic Black-Scholes has some assumptions which do not hold in real life and can lead to losses. Analysis of the continuous trading assumption is presented in [2, Emanuel Derman], [3, Derman and Taleb] and [5, Haug and Taleb]. Analysis of transaction costs is available in [9, Hayne Leeland]. Analysis of continuous dividend yield is presented in [9, Ralf and Rogers].

A case we haven't found (which doesn't mean it wasn't studied, only that we haven't found it and therefore derived the conclusions independently) is when Black-Scholes is used to price options on a stock index but the replicating portfolio uses index futures due to the unavailability or difficulty of trading directly in the index, as required by the model. A very brief paper touching the subject (incorrectly, in our opinion) can be found online as [1, Antonie Kotze], at least proving that the issue is known.

So we arrive at the task of deriving the model ourselves, which is what this paper is about. In case we re-discovered the wheel, we stress again that we arrived at the results independently, which reminds us of two quotes (Google will reveal the authors): "What I cannot create, I do not understand" and "Don't reinvent the wheel, unless you plan on learning more about wheels".

\section{References}

[1] Antonie Kotze, Delta Hedging: Futures Versus Underlying Spot, quantonline.co.za.

[2] Emanuel Derman, When You Cannot Hedge Continuously, Goldman Sachs Quantitative Strategies Research Note.

[3] Emanuel Derman, Nassim Nicholas Taleb, The Illusions of Dynamic Replication, Quantitative Finance, Vol. 5, No. 4, August 2005, 323-326.

[4] Espen Gaarder Haug, The Complete Guide To Option Pricing Formulas, McGraw-Hill, 1998.

[5] Espen Gaarder Haug, Nassim Nicholas Taleb, Option Traders Use (very) Sophisticated Heuristics, Never the Black-Scholes-Merton Formula, Journal of Economic Behavior and Organization, Vol. 77, No. 2, 2011. 
[6] Fabrice Douglas Rouah, Four Derivations of the Black-Scholes Formula, www.frouah.com.

[7] Hayne Leeland, Option Pricing and Replication with Transaction Costs, Journal of The Journal of Finance, Vol 40, No. 5 (Dec 1985), 1283-1301.

[8] John Hull, Options, Futures and Other Derivatives, Prentice Hall, 6nd edition, 2005.
[9] Ralf Korn, Leonard Rogers, Stocks Paying Discrete Dividends: Modelling and Option Pricing, The Journal of Derivatives, Winter 2005, Vol. 13, No. 2: pp. 44-48.

[10] Salih Neftci, An Introduction to the Mathematics of Financial Derivatives, Academic Press, 2nd edition, 2000.

[11] Thomas Björk, Arbitrage Theory in Continuous Time, Oxford Finance, 2nd edition, 2004. 